\title{
Rock and Soil Damage-Fracture Space Mechanics: the Divisional Damage-Fracture Theory
}

\author{
Faning Dang ${ }^{1}$ Houqun Chen ${ }^{1,2}$ Weihua Ding ${ }^{1}$ Xiaotao Yin ${ }^{1,3}$ \\ ${ }^{1}$ Xi'an University of Technology, Xi'an 710048, P. R. China \\ ${ }^{2}$ China Institute of Water resources and Hydropower Research, Beijing 100044, P. R. China \\ ${ }^{3}$ Institute of Rock and Soil Mechanics, Chinese Academy of Sciences, Wuhan 430071, P. R. China
}

\begin{abstract}
This article points out each of the current analytical methods used in CT mechanics of rock and soil avail of observation, wasting the precious quantitative information of CT datum. Based on the conception of the intercepted section in the damage-fracture space, utilizing the knowledge of the set theory, the measure theory and the Lebesgue integral, the rock and soil material is classified as the safety zone, the damaging zone and the fractured zone. The problems such as the upper and the lower boundary surface of the damaging zone, the relative length of the fractured zone, the number and the space size of the cracks in the fractured zone are studied. Meanwhile, the paper defining the rules of compressing and expanding, establishing the rock and soil mass constitutive relation of the divisional damage-fracture space, simultaneously furthering to deduce the basic equation of the rock and soil damage-fracture space theory. If the damaging zone is merged into the safety zone or the fractured zone, the constitutive relation of the divisional damagefracture space degenerates into breakage mechanics for geological materials presented by zhujiang Shen academician.
\end{abstract}

Keywords: The damage-fracture ratio, The damagefracture space, The safety zone,The damaging zone, The fractured zone, The constitutive relation of the divisional damage-fracture space, The rock and soil damage-fracture space theory

\section{Preface}

The CT method can examine the inner density change of a material or structure by not to damage it, its characteristics are that the experimental expenditure is large and that the quantitative information of each point can be presented. The CT datum is rare resources because of its rarity and the abundant information contained. Therefore it should be utilized sufficiently. However, the current research on the evolution process of the damage and meso-crack is observed and analyzed only from the view of the size, mean and variance of the $\mathrm{CT}$ number, the gray variety of the $\mathrm{CT}$ image[1]-[4], the statistic frequency of the CT number[5], the damage variable[5], the damage evolution equation[6], the damage evolution rate[7], the damage contour line[8], the threshold value of the CT number[9], the structural tensor of the soil mass ${ }^{[9]}$ and the density damaged increment[10] etc., a systematic and characteristic researching method has not formed so far. The characteristics of these methods are that the quantitative information of CT resolving power elements averaged the mean information, applying the rare quantitative information to the qualitative description, all these result in wasting the resources of the CT image. On the other hand, the direct observation of the $\mathrm{CT}$ image generates the error of the visual angle. The same kind of the research begins early [11]-[12], but the research methods are the same to the above, without big breakthrough ${ }^{[13-14]}$. So the CT research of the current rock and soil mechanics actually locates at "the observation stage of the CT image".

Article[15] defines the concepts of the perfect degree and the damage-fracture degree of a certain point in the rock and soil medium space, the $\lambda$ level perfect field and the damage-fracture field, the $\lambda_{1}-\lambda_{2}$ level intercepted sector etc., and studies some of their basic quality. Article[16] utilizing the knowledge of the set theory and the measure theory defines the conceptions such as the $\lambda$ level damage-fracture ratio and the damage-fracture rate, the $\lambda_{1}-\lambda_{2}$ level intercepted sector ratio and rate, the $\lambda$ level damage-fracture content on rock, furthers to research the relation among the $\lambda$ level damage-fracture rate, the CT number and the density, the relation among the bulk strain, the density damaged increment and the CT number, the relation among the strain, the stress and the density damaged increment etc. .

In this paper, according to the damage-fracture space theory and the physical state indexes theory of "the rock and soil damage-fracture space mechanics", the rock and soil material is classified as the safety zone, the damaging zone and the fractured zone by utilizing the knowledge of the set theory and the 
concept of the intercepted sector, presenting the definition of the rules of compressing and expanding, constructing "the divisional damage-fracture constitutive relation" of the rock and soil mass, furthering to deduce the basic equation of the rock and soil damage-fracture space theory. If the damaging zone is not considered, namely merged into the safety zone or the fractured zone, the constitutive relation of the divisional damage-fracture degenerates to the rock and soil breakage mechanics theory. If the fractured zone is not considered, the constitutive relation of the divisional damage-fracture degenerates to the rock and soil damage mechanics theory. Certainly, according to the theory in this paper, the physical zones of the material located in can be classified as more and more detailed, making it be more applicable to the real condition of the rock and soil material. The more nearer to the actual situation the mode is, the more difficulty to operate it is, so the material is only divided into three zones here.

This paper establishes the core content of the rock and soil damage-fracture space mechanics, the application pertinent to this theory model will be discussed in another paper. The theory in this paper builds the base of the uniform description of the mesomechanics and the macro-mechanics, and realizes the uniform description of the continuum mechanics and porous media or crannied media mechanics.

The method of this article suites not only for the rock mass material but also for the soil mass material, but the following examples regard rock material as the object described, simultaneously the following discussing will be confined to the damage-fracture space, the conceptions which are not explained here can refer to the reference [15],[16].

The conceptions not explained the derivation in this paper all are independently originated by author, all belong to the original job.

\section{The position of the damage- fracture produced}

As far as a certain state is concerned, the position of the meso-crack sprouted should happen about the part where the value of $d(x, y, z)$ is maximum, this zone can be presented by the set as the following,

$$
\left\{\left(x_{0}, y_{0}, z_{0}\right) \mid d\left(x_{0}, y_{0}, z_{0}\right) \geq\left(d_{\max }-\varepsilon\right)\right\}
$$

in which $d_{\max }=\max \{d(x, y, z) \mid(x, y, z) \in \Omega\}$, and $\varepsilon$ is a suitable small real number. These have already been proved by the test results ${ }^{[2]}$.

As far as a certain uploading procedure is concerned, the position of the meso-crack sprouted should take place about the part where the value of the density damaged increment is maximum, namely the place about maximum of the density altered, this zone can be presented by the set as the following,

$\left\{\left(x_{0}, y_{0}, z_{0}\right) \mid \Delta D\left(x_{0}, y_{0}, z_{0}\right) \geq\left(\Delta D_{\max }-\varepsilon\right)\right\}$

in which $\Delta D_{\max }=\max \{\Delta D(x, y, z) \mid(x, y, z) \in \Omega\}$, and $\varepsilon$ is a suitable small real number.

The position of the meso-crack sprouted is described from angles of a certain sate and a certain procedure respectively, these two methods are identical to each other in the space distribution, which has already been proved by lots of test results derived of Xiurun Ge academician ${ }^{[2]}$ and Gengshe Yang ${ }^{[5]}$.

\section{The criterion of the damage- fracture produced}

Whether the damage-fracture can be produced or not at certain point, the density value and the variation of the density at the point can be used as the criterion. Supposed the damage-fracture degree of the cement of a certain kind of rock is $d_{(0)}$, the size of the macrocrack is a constant number for a specific kind of rock, so there exist two positive constants $\varepsilon_{f}$ and $\varepsilon_{d}$, such that

the material at this point has fallen into fractured when $d_{(1)}(x, y, z)-d_{(0)}>\varepsilon_{f}$,

the material at this point is damaging when $\varepsilon_{f} \geq d_{(1)}(x, y, z)-d_{(0)} \geq \varepsilon_{d}$,

the material at this point locates in the safety situation when $d_{(1)}(x, y, z)-d_{(0)}<\varepsilon_{d}$.

Where, $d_{(1)}(x, y, z)$ represents the damagefracture degree of a certain point in the rock sample.

If the CT number of the cement equates or is very near to the maximum $\mathrm{CT}$ number $(\max H)$ of the rock sample, then $d_{(0)} \approx 0$, the above criterion turns into the following,

the material at this point has fallen into fractured if $d_{(1)}(x, y, z)>\varepsilon_{f}$,

the material at this point is damaging if $\varepsilon_{f} \geq d_{(1)}(x, y, z) \geq \varepsilon_{d}$,

the material at this point locates in the safety situation if $d_{(1)}(x, y, z)<\varepsilon_{d}$.

\section{The safety zone, the damaging zone and fractured zone}


According to the above criterion, a space of the rock sample is classified as the safety zone, the damaging zone and the fractured zone. Their definitions are expressed by the following respectively,

$$
S=\left\{(x, y, z) \mid d(x, y, z)-d_{(0)}<\varepsilon_{d}\right\} \text { is called }
$$

the safety zone of the rock sample,

$$
D=\left\{(x, y, z) \mid \varepsilon_{d} \leq d(x, y, z)-d_{(0)} \leq \varepsilon_{f}\right\} \quad \text { is }
$$

called the damaging zone of the rock sample,

$$
F=\left\{(x, y, z) \mid d(x, y, z)-d_{(0)}>\varepsilon_{f}\right\} \text { is called }
$$

the fractured zone of the rock sample.

Ditto, if the CT number of the cement equates or is very near to the maximum CT number $(\max H$ ) of the rock sample, then $d_{(0)} \approx 0$, the above zones turns into the following: The set of the safety zone $S$ is practically a $\varepsilon_{d}$ level non-damage-fracture field. The set of the damaging zone $D$ is a $\varepsilon_{d}-\varepsilon_{f}$ level intercepted section. The set of the fractured zone $F$ is a strong $\varepsilon_{f}$ level damage-fracture field. Actually, these are three different level intercepted sections.

Obviously, $\varepsilon_{f}$ and $\varepsilon_{d}$ are two material constants, which are determined by the physical property of the rock, are the boundary points of the rock among the different physical states, are called the upper and lower factors of the material's damaging zone respectively.

In this way, the three states in which the rock sample locate are actually defined, ditto the rock sample can be classified as the more detailed sate zones.

\section{The upper and the lower boundary surfaces of the damaging zone}

The interfaces among the safety zone, the damaging zone and the fractured zone are defined by using the points set. They are not the simple curve or curved surface, mix with each other, some isolated points exist contemporarily. So the two boundary surfaces among the three zones are necessary to define from the angle of the set theory.

If the any $\delta \mathrm{CT}$ scale neighborhood at the point $\left(x_{0}, y_{0}, z_{0}\right)$ contains the points of the safety and damaging zones at the same time, then this point $\left(x_{0}, y_{0}, z_{0}\right)$ is called the lower boundary point of the damaging zone, all of the lower boundary points constitute a set called the lower boundary surface of the damaging zone, which is denoted by $b_{\varepsilon_{d}}$.
Ditto, If the any $\delta \mathrm{CT}$ scale neighborhood at the point $\left(x_{0}, y_{0}, z_{0}\right)$ contains the points of the fractured zone and the points of the damaging zone or safety zone, then this point $\left(x_{0}, y_{0}, z_{0}\right)$ is called the upper boundary point of the damaging zone, all of the upper boundary points form a set called the upper boundary surface of the damaging zone, which is denoted by $b_{\varepsilon_{f}}$. The upper boundary surface is also called the boundary surface of the crack.

Here the so-called any $\delta \mathrm{CT}$ scale neighborhood about a point indicates the scale of the $\delta$ neighborhood (the side length or the diameter) which should be greater than the resolving power elements scale of the $\mathrm{CT}$. Here the so-called surface is only for the purpose of matching with customarily, which is actually a set of points and not a surface. Moreover, the points named here actually equals the point of the CT scale, which has the dimensional size and don't be a point defined in the mathematics.

Moreover, the following should be noticed, the category of any CT scale neighborhood $\delta\left(x_{0}, y_{0}, z_{0}\right)$ at the boundary point $\left(x_{0}, y_{0}, z_{0}\right)$ may belong to the following: ( a) the neighborhood includes the points of the safety zone and the damaging zone at the same time, ( b) the neighborhood includes the points of the damaging and the fractured zones simultaneously, (c) the neighborhood includes the points of the safety and the fractured zones simultaneously, (d) the neighborhood includes the points of the safety zone, the damaging zone and the fractured zone at the same time. The upper boundary surface contains (b), (c) and (d) three conditions simultaneously.

\section{The measurement of the zones and the boundary surfaces}

The sizes of the safety, the damaging and the fractured zones can be estimated by measure. About definition of the measure, please referring to the measure theory ${ }^{[17]}$. The measures of the safety, the damaging and the fractured zones can be denoted by $m(S), m(D)$ and $m(F)$.

The sizes of the lower and upper boundary surfaces can be estimated by measure too. Their measures can be denoted by $m\left(b_{\varepsilon_{d}}\right)$ and $m\left(b_{\varepsilon_{f}}\right)$.

\section{The relative length of the fractured zone}


The same measure of the different fractured zones may have a variety of the shape, from the mechanics view its lengths are noticed, the lengths of the fractured zones with the same area can be estimated by the ratio between measure $m\left(b_{\varepsilon_{f}}\right)$ and $m(F)$. That is to say, the measure $m\left(b_{\varepsilon_{f}}\right)$ of the upper boundary surface of the damaging zone is divided by the measure $m(F)$ of the fractured zone, it is called the relative length of the fractured zone, which is denoted by $l_{d}$, namely

$$
l_{d}=\frac{m\left(b_{\varepsilon_{f}}\right)}{m(F)}
$$

The larger the relative length of the fractured zone is, the narrower and longer the macro-crack is, otherwise macro-crack is wider and shorter.

The rock sample in the loading processing generates the cracks, at the early stage the cracks length gradually increasing with the increasing outside force, the relative length $l_{d}$ of the fractured zone increases gradually, too. With the cracks development in the rock sample, the cracks begin to widen and the area of the fractured zone increases, too, its relative length decreases at the same time. Therefore, $l_{d}$ increases in early stage and decreases afterwards, it reflects the expansive process of the cracks inside the rock sample that develops in the direction of the length previously and heads for the direction of the width afterwards.

The relative length of the fractured zone is a relative physical parameter which can measure the length of the cracks with the same area, its minimum value is presented as following,

$$
l_{d \min }=\frac{4}{l}
$$

There-into, $l$ is the actual length of the fractured zone. The relative length is not the true length of the crack. Moreover, the relative length is not only a relative length of a certain crack, but also the relative length of a crack family.

\section{The quantity and the space size of the cracks}

From the above discussion, it is concluded that the fractured zone is actually a crack family, the cracks quantity of the fractured zone is defined by the theory of the connected set in the mathematics.

If the arbitrary two points inside a space set can be connected by a random folding-line within the set, then it is called connected set, else is named disconnected set. A connected set within the fractured zone is labeled a piece of crack.

The quantity of the disconnected subsets within the fractured zone of the rock sample is presented the number of the cracks inside the fractured zone.

\section{The space size of the single crack}

For a piece of crack within the fractured zone, recorded as $d_{\lambda}^{(i)},(i=1,2, \cdots, n)$, where the $n$ is the quantity of the cracks, it is actually a connected subset, the minimum value and maximum value of the three coordinates of the points within the connected subset are denoted by $x_{\min }, y_{\min }, z_{\min }$ and $x_{\max }, y_{\text {max }}, z_{\text {max }}$ respectively, then the effective space size of the crack is defined as following

$$
l=\left[\left(x_{\max }-x_{\min }\right)^{2}+\left(y_{\text {max }}-y_{\text {min }}\right)^{2}+\left(z_{\text {max }}-z_{\text {min }}\right)^{2}\right]^{\frac{1}{2}}
$$

The relative length of the fractured zone $l_{d}$ is different from the concept of the effective space size of the crack. The former reflects the fragmentation degree of the rock sample, but the latter reflects the space size of the checked surface of the rock sample. Such as the figure 1 presents that the effective space sizes of the two cracks within sub-figure (a) and (b) is equivalency but they differ much in the relative length of the fractured zone.

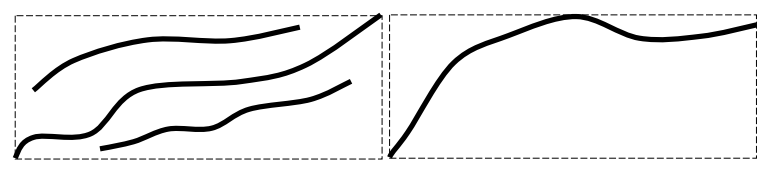

(a)

(b)

Fig. 1: The sketch map of the cracks.

\section{The rules of compressing and expanding}

When the stress states of a certain point in the rock change from $\sigma_{i j}$ to $\sigma_{i j}+\Delta \sigma_{i j}$, the damage-fracture degrees correspondingly change from $d_{(1)}(x, y, z)$ to $d_{(2)}(x, y, z)$,

the point is called the compressing state if $d_{(2)}(x, y, z)-d_{(1)}(x, y, z)>0$.

the point is called the expanding state if $d_{(2)}(x, y, z)-d_{(1)}(x, y, z)<0$. It is called unloading expanding when the load is decreasing, and it is called shear expanding when the shear stress is increasing. 
the point is called the neutral state if $d_{(2)}(x, y, z)-d_{(1)}(x, y, z)=0$.

It is noticeable that the compressing state defined here equate the increasing of the loads, but the expanding state defined here doesn't equate the decreasing of the loads. The material tend to safe when it is in unloading expanding, and tend to failure when it is in shear expanding.

If the material locates in the shear expanding state, the safety zone constantly translates into the damaging zone, while the damaging zone ceaselessly changes into the fractured zone.

\section{The divisional description of the rock and soil constitutive relation}

Previously, the variety of the constitutive relations have been established. The common features of all the constitutive relation established are according to the different stress states in which the material locates. Because the rock and soil material in the same stress level can correspond to many different deformation states, this kind of constitutive relation must differentiate the material stages such as firstly uploading, unloading, uploading again, unloading again, the times of the uploading and unloading, the stage when the material locates in the hardening or the softening. In this way, the constitutive relation of the rock and soil material becomes exceptionally complex, and bringing many difficulties in calculating and applying.

In the following, the divisional damage-fracture constructive relation of the rock and soil is established according to the state zones where the material locate in the above paragraph.

As to the safety zone $S$ of the material, the elastic constitutive relation is adopted as the following,

$$
\sigma_{e}=E \varepsilon_{e}
$$

As to the damaging zone $D$ of the material, the damage constitutive relation is adopted as the following,

$$
\sigma_{d}=\alpha_{1}(1-d) E \varepsilon_{d}=\alpha_{1} p E \varepsilon_{d}
$$

As to the fractured zone $F$ of the material, the force which can bear is small, moreover the deformation is hard to resume, the plastic constitutive relation is adopted as the following,

$$
\sigma_{p}=\alpha_{2} D_{p} \varepsilon_{p}
$$

Where, $p$ and $d$ present the perfect degree and the damage-fracture degree of a certain point in the rock sample, $\alpha_{1}$ and $\alpha_{2}$ represent the variable coefficients of the material.
The divisional damage-fracture constitutive relation of the rock and soil material has the simple form and the clear physical meaning etc..

\section{The constitutive theory of the rock and soil damage-fracture space}

This article regards the rock sample as the material being composed of the safety zone, the damaging zone and the fractured zone. In fact, they are the $\varepsilon_{d}$ level non-damage-fracture field, the $\varepsilon_{d}-\varepsilon_{f}$ level intercepted section and the strong $\varepsilon_{f}$ level damage-fracture field respectively, which are three different level intercepted sections. Supposed their intercepted section rates are $D_{n}^{0-\varepsilon_{d}}, D_{n}^{\varepsilon_{d} \varepsilon_{f}}$ and $D_{n}^{\varepsilon_{f}^{-1}}$ respectively, the total average stress and average strain can be expressed as the following,

$$
\begin{aligned}
& \bar{\sigma}=D_{n}^{0-\varepsilon_{d}} \bar{\sigma}_{e}+D_{n}^{\varepsilon_{d}-\varepsilon_{f}} \bar{\sigma}_{d}+D_{n}^{\varepsilon_{f}-1} \bar{\sigma}_{p} \\
& \bar{\varepsilon}=D_{n}^{0-\varepsilon_{d}} \bar{\varepsilon}_{e}+D_{n}^{\varepsilon_{d}-\varepsilon_{f}} \bar{\varepsilon}_{d}+D_{n}^{\varepsilon_{f}-1} \bar{\varepsilon}_{p}
\end{aligned}
$$

In the formulas, the subscripts such as $e, d, p$ represent the safety zone, the damaging zone and the fractured zone respectively. The total average stress, the total average strain and the corresponding stresses and strains in the three zones can be determined by the following formulas,

$$
\begin{array}{ll}
\bar{\sigma}=\frac{1}{m(\Omega)} \int_{\Omega} \sigma \mathrm{d} \Omega, & \bar{\sigma}_{e}=\frac{1}{m(S)} \int_{S} \sigma_{e} \mathrm{~d} S, \\
\bar{\sigma}_{d}=\frac{1}{m(D)} \int_{D} \sigma_{d} \mathrm{~d} D, & \bar{\sigma}_{p}=\frac{1}{m(F)} \int_{F} \sigma_{p} \mathrm{~d} F \\
\bar{\varepsilon}=\frac{1}{m(\Omega)} \int_{\Omega} \varepsilon \mathrm{d} \Omega & \bar{\varepsilon}_{e}=\frac{1}{m(S)} \int_{S} \varepsilon_{e} \mathrm{~d} S, \\
\bar{\varepsilon}_{d}=\frac{1}{m(D)} \int_{D} \varepsilon_{d} \mathrm{~d} D, & \bar{\varepsilon}_{p}=\frac{1}{m(F)} \int_{F} \varepsilon_{p} \mathrm{~d} F
\end{array}
$$

Where, $m(*)$ presents the measure of the $\operatorname{set}(*)$. Certainly, the integral here is Lebesgue integral.

The conceptions of the measure and the Lebesgue integral can be consulted in the article ${ }^{[17]}$.

The local strain coefficients of the safety zone, the damaging zone and the fractured zone can be respectively defined as the following,

$$
\beta_{e}=\frac{\bar{\varepsilon}_{e}}{\bar{\varepsilon}}, \beta_{d}=\frac{\bar{\varepsilon}_{d}}{\bar{\varepsilon}}, \beta_{p}=\frac{\bar{\varepsilon}_{p}}{\bar{\varepsilon}}
$$

replacing the corresponding parts in the formula (4), the divisional damage-fracture constitutive relation can draw the following, 


$$
\begin{aligned}
\bar{\sigma} & =D_{n}^{0-\varepsilon_{d}} \bar{\sigma}_{e}+D_{n}^{\varepsilon_{d}-\varepsilon_{f}} \bar{\sigma}_{d}+D_{n}^{\varepsilon_{f}-1} \bar{\sigma}_{p} \\
& =D_{n}^{0-\varepsilon_{d}} D_{e} \bar{\varepsilon}_{e}+D_{n}^{\varepsilon_{d}-\varepsilon_{f}} D_{d} \bar{\varepsilon}_{d}+D_{n}^{\varepsilon_{f}-1} D_{p} \bar{\varepsilon}_{p} \\
& =D_{n}^{0-\varepsilon_{d}} \beta_{e} D_{e} \bar{\varepsilon}+D_{n}^{\varepsilon_{d}-\varepsilon_{f}} \beta_{d} D_{d} \bar{\varepsilon}+D_{n}^{\varepsilon_{f}-1} \beta_{p}
\end{aligned}
$$

so, the formula below exists,

$\bar{\sigma}=\bar{D} \bar{\varepsilon}$

the $\bar{D}$ in the last formula (6) can be presented as the following formula,

$$
\bar{D}=D_{n}^{0-\varepsilon_{d}} \beta_{e} D_{e}+D_{n}^{\varepsilon_{d}-\varepsilon_{f}} \beta_{d} D_{d}+D_{n}^{\varepsilon_{f}-1} \beta_{p} D_{p}
$$

where $D_{e}, D_{d}$ and $D_{p}$ present the elastic constant matrix, the damage matrix and the plastic matrix of the divisional constitutive relation respectively, namely

$$
D_{e}=E, D_{d}=\alpha_{1}(1-d) E=\alpha_{1} p E, D_{p}=\alpha_{2} D_{p}
$$

in these formulas, $p$ and $d$ present the perfect degree and the damage-fracture degree of a certain point in the rock sample, $\alpha_{1}$ and $\alpha_{2}$ represent the variable coefficients.

If lets $\sigma_{e}=D_{e} \bar{\varepsilon}, \sigma_{d}=D_{d} \bar{\varepsilon}$ and $\sigma_{p}=D_{p} \bar{\varepsilon}$, the formula below is the basic equation of the rock and soil damage-fracture space theory.

$\bar{\sigma}=D_{n}^{0-\varepsilon_{d}} \beta_{e} \sigma_{e}+D_{n}^{\varepsilon_{d}{ }^{-} \varepsilon_{f}} \beta_{d} \sigma_{d}+D_{n}^{\varepsilon_{f}-1} \beta_{p} \sigma_{p}$

$\sigma_{e}, \sigma_{d}$ and $\sigma_{p}$ in the formula (7) have not been the average stresses yet, they are the stresses at each points.

If the damaging zone is not considered, namely which is merged into the safety zone or the fractured zone, the rock and soil damage-fracture space theory degenerates into breakage mechanics for geological material presented by zhujiang Shen academician ${ }^{[18]}$.

\section{Conclusions}

According to the concepts of the damage-fracture degree, the damage-fracture space and the intercepted section etc. which are defined in this paper and the article ${ }^{[15,16]}$, the rock and soil material is classified as the safety zone, the damaging zone and the fractured zone based on the knowledge of the set theory, the measure theory and the Lebesgue integral etc., the problems such as the upper and the lower boundary surface of the damaging zone, the relative length of the fractured zone, the number and the space size of the cracks in the fractured zone are studied, then the paper presenting the definition of the compressing and expanding rule, establishing the divisional constitutive relation of the rock and soil mass, simultaneously furthering to deduce the basic equation of the rock and soil damage-fracture space theory. If the damaging zone merges into the safety zone or the fractured zone, the constitutive relation of the divisional damagefracture space degenerates to breakage mechanics for geological material of zhujiang Shen academician's.

$D_{p} \bar{\varepsilon}$ The theory in this paper is suitable for the mesomechanics and macro-mechanics, for the continuum mechanics and porous media or crannied media mechanics at the same time.

\section{Acknowledgement}

This project was supported by the National Natural Science Foundation of China (No. 50679073 and 90510017)

\section{References}

[1] Kawakata H., Cho A. and T. Kiyama et al., Three-dimensional observations of faulting process in Westerly granite under uniaxial condition by CT X-ray scan. Tectonophysic, 313:293-305,1999.

[2] X.R. Ge, J.X. Ren and Y.B. Pu et al, Primary study of CT real-time testing of fatigue mesodamage propagation law of rock, Chinese Journal of Geotechnical engineering, 23(2): 191 195, 2001.

[3] Phillips D.H., Lannutti J. J., Measuring physical density with X-ray computed tomography, NDT\&E International,30(6):339-350, 1977.

[4] Shi B., Y. Murakami, Z. Wu et al, Monitoring of internal failure evolution in soils using computerization X-ray topography. Engineering Geology, 54:321-328, 1999.

[5] G.S. Yang, D.Y. Xie and C.Q. Zhang et al, CT identification of rock damage properties, Chinese Journalof Rock Mechanics and Engineering, 15(1):48 54, 1996.

[6] Z.H. Lu, Z.H. Chen, Y.B. Pu, A CT study on the crack evolution of expansive soil during drying and wetting cycles, Chinese Rock and Soil Mechanics,23(4):417 422, 2002.

[7] H. Jian, S.C. Li, W.S. Zhu, L.F. Qi, CT analyses of brittle materials under hydraulic pressure and uniaxial compression, Chinese Rock and Soil Mechanics,23(5):587 591, 2002.

[8] Y.S. Chen, N. Li, A.G. Li and Y.B. Pu et al, Analysis on meso-damage process of noninterpenetraed jointed media by using CT, Chinese journal of Rock Mechanics and Engineering, 19(6):702 706, 2000.

[9] X.J. Li, D.L. Zhang, Monitoring change of structure of road foundation soil in uniaxial 
compression test with CT, Chinese Journal of geotechnical engineering, 22(2):205 209, 2000.

[10] W.H. Ding, Y.Q. Wu, Y.B. Pu et al, measurement of crack width in rock interior based on X-ray CT, Chinese Journal of Rock Mechanics and Engineering, 22(9):1421 1425, 2003.

[11] Segal E., Notea A., Segal Y., Dimensional information through industrial computerized tomography. Materials Evaluation, 40:1268-1272, 1982.

[12] Takashi I., Koichi K. and Yuichiro M. et. al., Preliminary study on application of X-radial CT scanner to measurement of void fractions in steady state two-phase flows, Journal of Nuclear Science and Technology, 20(1):1-12, 1983.

[13] Kawakata H., Cho A., and T.Kiyama et al. Threedimensional observations of faulting process in Westerly granite under uniaxial conditions by CT X-radial scan. Technophysics (313): 293-305, 1999.

[14] K. Ueta, K. Tani, T. Kato. Computerized X-radial topography analysis of three-dimensional fault geometries in basement-induced wrench faulting. Engineering Geology, 56:197-210, 2000.

[15] F.N. Dang, Rock and soil damage-fracture space mechanics $*(1)$, Damage-fracture space, International Conference on Intelligent Systems and Knowledge Engineering,2007.

[16] F.N. Dang, Rock and soil damage-fracture space mechanics *(2), Physical state indexes, International Conference on Intelligent Systems and Knowledge Engineering,2007.

[17] Halmos, P. R., Measure Theory, Van Nostrand, New York, 1968.

[18] Z.J. Shen, Breakage mechanics for geological material: an ideal brittle-elastic-plastic model,
Chinese Journal of geotechnical engineering, 25(3):253-257, 2003. 